\title{
VAMOS COMUNICAR? \\ Revisão Teórica sobre a Relação entre a Comunicação Interna nas Organizações e a Motivação dos Colaboradores
}

\author{
Sabrina Lourenço de Almeida ${ }^{1}$ \\ Filomena Capucho ${ }^{2}$ \\ Paulo Ribeiro ${ }^{3}$
}

\begin{abstract}
Resumo: As organizações têm vindo a sofrer diversas alterações, sendo que a forma como os indivíduos são vistos passou de uma perspetiva de mão-de-obra ou trabalhador para uma abordagem de colaborador. No seio de uma organização, todos os indivíduos comunicam naturalmente entre si, tornando-se impossível não comunicar. A presença dum indivíduo, só por si, já transmite uma mensagem para o mesmo ou para os outros. Mesmo quando nada é dito, está-se a transmitir que não se pretende comunicar, e esta ação só por si já se traduz em comunicação.

Relativamente à definição de comunicação, opomos uma definição mecanicista, que essencialmente se resume a um indivíduo que emite a mensagem a outro através de um canal (modelo de Jakobson, 1969) a um modelo de orquestra, no qual a comunicação é definida por relações interpessoais que se estabelecem em contextos sociais (Goffman, 2002; Watzlawick, 2011).

A par destas mudanças, a atuação dos colaboradores no interior das organizações tem sofrido alterações, reconhecendo-se a importância da motivação dos mesmos para o sucesso da organização. Por seu turno, estudos recentes relacionam a qualidade da comunicação organizacional com o êxito organizacional, que, por sua vez, também está relacionado com a motivação dos colaboradores. Existem diversas teorias motivacionais, que essencialmente se dividem em três grandes grupos: conteúdo, processo e resultado.

Em síntese, a organização é composta por indivíduos que comunicam entre si e criam relações. Estas relações são fundamentais para se poder falar em motivação, pois dificilmente
\end{abstract}

\footnotetext{
${ }^{1}$ Grupo Visabeira. Email: sa_bsi@hotmail.com

${ }^{2}$ Universidade Católica Portuguesa -Viseu. Email: fcapucho@gmail.com

${ }^{3}$ Universidade Católica Portuguesa -Viseu Email: pribeiro@viseu.ucp.pt
} 
alguém consegue estar motivado se não se sentir bem no seu local de trabalho.

O principal objetivo deste trabalho é a apresentação dos resultados da leitura crítica de bibliografia de fundamentação teórica para o estudo da relação entre a comunicação interna das organizações e a motivação dos colaboradores.

Palavras-chave: Organização, Comunicação, Motivação.

\begin{abstract}
Organizations have suffered changes in recent years and the way how individuals are viewed has passed from a vision of labor or worker to a collaborative approach. Inside the organization, all individuals naturally communicate with each other, making it impossible not to communicate. The simple presence of an individual already transmits a message to himself or to others. Even when individuals don't say anything, they transmit that they have no intention to communicate, and this action is already a form of communication.

Regarding the definition of communication, we oppose a mechanistic view, which is essentially defined as an individual who sends a message through a channel to another individual (model of Jakobson, 1969), to an orchestra model, in which communication is defined as a creation of interpersonal relations, which are established in social contexts (Goffman, 2002, Watzlawick, 2011).

Because of all the changes which have occurred in organizations, the way how employees function inside organizations has suffered modifications, and the way how their motivation areis viewed for the organization success has gained emphasis. In turn, recent studies relate the role of organizational communication on organizational success, which is also related to employee's motivation. There are several motivational theories, which fall essentially into three large groups: content, process and outcome.

Concluding, the organization is formed by individuals who communicate with each other and create relationships. These relationships are essential to motivation, because you can hardly be motivated if you do not feel good in your workplace.

The main objective of this work is the presentation of the results from a critical review of literature on the study of a relationship between the internal communication of organizations and motivation of collaborators.
\end{abstract}

Keywords: Organization, Communication, Motivation 


\section{A ORGANIZAÇÃO}

Atualmente vivemos num mundo globalizado, em que tudo existe em todo o lado, e tudo se transmite e espalha com muita facilidade e rapidez, mas nem sempre foi assim. Se recuarmos até ao início do século passado, percebemos que a forma de organização das sociedades e a própria forma de atuação das organizações mudou consideravelmente. Rapidamente se percebeu que os seres humanos são delicados e difíceis de gerir, uma vez que existe um conjunto de características que os diferenciam, nomeadamente as competências, aptidões, preferências, motivações e estilos (Ferreira, Martinez, Nunes \& Duarte, 2015).

Se pesquisarmos o significado da palavra organização em fontes mais clássicas como os dicionários, verificamos que as características apontadas são: planeamento; disposição; estrutura; ordenação; composição; organismo; ordem; eficiência; coordenação; coerência que por sua vez forma um todo. O substantivo deriva do verbo organizar, cuja raiz etimológica vem do latim «organu» (Bilhim, 1996; Drosdowski, 1989; Hornby, 2000; Porto Editora, 2008; Robert, 2001).

Podemos definir a organização como um lugar físico ou abstrato, constituído por pessoas e que estabelecem relações entre si (Pereira, 2004; Souza \& Santos, 2014; Teixeira, 2005). Desta forma, o fator humano assume um papel central, pois é este que desenvolve os esforços para a obtenção dos objetivos organizacionais propostos (L. A. S. Almeida, 2013). Estes objetivos podem ser de natureza pública ou privada e lucrativa ou não, sendo que para tal é imprescindível fazer uso da comunicação (Machado, Machado \& Portugal, 2014; Pinto, 2013).

Torna-se fundamental que a organização alinhe o planeamento dos recursos humanos com o planeamento dos negócios para garantir o alcance dos resultados desejados (Nascimento, Marinho, Ramos, Santos, Guimarães \& Meneze, 2014). Neste sentido, a gestão dos recursos humanos necessitou de alargar a sua área de atuação, passando a desempenhar um vasto leque de funções, que não se restringem apenas às fases de seleção e recrutamento dos indivíduos (Barracho, 2014).

Finalizando, poderemos referir que os modelos tradicionais de gestão são lentos e não geram incentivos à inovação, que é atualmente uma das forças motoras de mercado. Logo, uma organização deve ter sempre em consideração o seu desempenho, mas também o seu ambiente sociocultural e sociopolítico, de forma a melhor abordar todas as mudanças que se possam registar (Vanderley, 2001). 


\subsection{Estrutura Organizacional}

Todas as organizações têm uma estrutura que pode estar melhor ou pior definida Para que as organizações possam alcançar os objetivos a que se propõem têm necessidade de escolher a estrutura que melhor se apropria (Cunha, Rego, Cunha \& Cabral-Cardoso, 2007; Teixeira, 2005). As funções no interior da organização reagrupam-se segundo semelhanças encontradas, com base num conjunto de critérios anteriormente definidos (Teixeira, 2005). A agregação de funções pode ser explicada em modelos mais complexos, nomeadamente através do modelo de Burns e Stalker (1961). Este modelo apresenta dois polos de sistemas organizacionais: mecanicista ou burocrático, normalmente adotados em ambientes estáveis e caracterizados pela definição de funções, métodos, responsabilidades e poder; orgânico, muitas vezes utilizado em ambientes de mudança, onde o contributo de conhecimento especializado e a partilha de experiências se torna essencial, sendo a tomada de decisão decentralizada.

As estruturas organizacionais são um conjunto de relações formais entre os diversos indivíduos que constituem a organização e que definem suas funções (Teixeira, 2005).

Sintetizando, as organizações têm evoluído e as estruturas organizacionais têm necessidade de se adaptar às novas mudanças, sendo importante notar que a comunicação desenvolve um papel fulcral no desenvolvimento da estrutura, pois os vários canais de comunicação permitem uma integração mais consistente das diversas partes (Kich \& Pereira, 2011).

\subsubsection{Departamentalização}

Associado ao conceito de estrutura empresarial surge inevitavelmente o termo de departamentalização. A departamentalização consiste num processo de agrupamento de funções semelhantes ou por atividades principais e que ocorre por diversas razões, nomeadamente: pelo volume de trabalho; agrupamento de funções similares; tradições e leis de trabalho; separação de funções de forma a evitar conflitos; necessidades de controlo de funções dissemelhantes (J. T. Oliveira, 2014; Teixeira, 2005).

Podemos apontar tanto efeitos positivos como negativos à departamentalização, pois esta parte da premissa da existência de uma boa relação entre os diversos departamentos; contudo, muitas vezes, acaba por se verificar um aumento de competitividade e conflitos de interesses entre estes (DeStefano, 2013). 
Os departamentos que se formam no interior da organização são de natureza horizontal - vários departamentos no mesmo nível hierárquico - e vertical - departamentos de níveis hierárquicos diferentes. Estes mesmos departamentos são criados com base num conjunto de critérios, dos quais se destacam a departamentalização por funções, produto, clientes, área geográfica, projetos, matricial ou formas combinadas (Teixeira, 2005).

\subsubsection{Tipos de Estrutura Organizacional}

Será, então, importante definir os tipos de estruturas organizacionais mais referidas na literatura desta área e mais adotadas pelas organizações:

- Estrutura simples - trata-se de uma estrutura muito comum em organizações familiares e de pequena dimensão, sendo normalmente constituída por dois níveis hierárquicos: gestor e colaboradores. A maioria das responsabilidades estão a cargo do gestor, não estando muitas vezes definidas de forma clara as tarefas e obrigações (S. Gomes, 2014; Teixeira, 2005).

- Estrutura funcional - é geralmente adotada quando já não é sustentável uma estrutura simples e caracteriza-se por uma delegação de autoridade e responsabilidade, criando departamentos de acordo com as funções clássicas da gestão: produção, comercial, financeiro, recursos humanos, marketing, entre outros (Chiavenato, 1995; S. Gomes, 2014; Teixeira, 2005).

- Estrutura divisionária - caracteriza-se por dividir as tarefas da organização de acordo com a diversidade dos seus produtos, serviços, mercados ou processos, sendo que cada uma das divisões criadas é independente e tem os seus especialistas funcionais. O gestor de cada divisão é responsável pela definição dos objetivos da sua equipa e pela colaboração com as restantes divisões da organização (Firmino, 2002; J. T. Oliveira, 2014; Teixeira, 2005).

- Estrutura matricial - trata-se de uma combinação da estrutura funcional e divisionária e é apropriada para organizações complexas (Teixeira, 2005).

- Estrutura por projetos - trata-se de um caso particular da estrutura matricial, pois diz respeito a uma divisão segundo um ou mais projetos (Teixeira, 2005). As divisões criadas terão naturalmente uma natureza temporária, mediante a duração do projeto a que se referem, a não ser que findo este surja um novo com características semelhantes (Firmino, 2002; Teixeira, 2005). 
- Estrutura por regiões - tem em consideração as regiões onde a organização se encontra, que podem ser de um mesmo país ou agrupando países diferentes; o elemento distintivo é a zona geográfica (Chiavenato, 1995; Firmino, 2002; J. T. Oliveira, 2014).

- Estrutura por Produto/Clientes/Processos - gere-se em função do número de produtos, dos critérios de segmentação de mercado, processos de produção (Chiavenato, 1995; Firmino, 2002; J. T. Oliveira, 2014).

- Estrutura em Rede - apresenta geralmente um único departamento central, que se caracteriza por ser o intermediário entre os restantes departamentos (Firmino, 2002; Teixeira, 2005).

Note-se que não existe uma estrutura que seja considerada universalmente a melhor escolha; por outras palavras, não existe nenhuma estrutura que seja melhor que outra, pois em cada caso específico, em cada contexto concreto, a escolha da estrutura é algo particular (S. Gomes, 2014). É igualmente verdade que numa mesma organização podem coexistir diversas estruturas (J. T. Oliveira, 2014).

\section{A COMUNICAÇÃO}

À semelhança dos outros animais, o Homem tem necessidade de se relacionar e interagir com os seus semelhantes, dispondo das mais diversas formas de o fazer. A comunicação liga os seres humanos em grupos, desde família, a amigos e conhecidos por todo o planeta (Santos, 2014). O indivíduo não consegue viver em sociedade sem comunicar (Schelles, 2008).

O ser humano distingue-se dos outros animais por ter acesso à fala; por outras palavras, os humanos são os únicos seres vivos com acesso a uma linguagem verbal. A fala caracteriza-se por ser um ato de vontade individual e de inteligência, que não seria possível sem a existência de uma língua comum a um conjunto de indivíduos (Francisco, 2012).

Para se poder falar e comunicar verbalmente torna-se necessário a partilha de significados de um conjunto de palavras, que mais não são do que uma representação simbólica do mundo referencial.

Na comunicação é fundamental que os símbolos tenham significados comuns entre os indivíduos envolvidos na interação; por outras palavras, é necessário que partilhem um mesmo código. Para além da comunicação verbal, existem várias outras formas de comunicação, sendo as mais comumente apresentadas a comunicação não-verbal e para-verbal. A comunicação não-verbal é a que está para além das palavras propriamente 
ditas, ou seja, acontece quando há interação com ou sem a utilização de palavras, isto é, percebe-se as expressões faciais, gestos, disposições dos objetos/ambiente, vestuário e as posturas corporais (J. G. Neves, Garrido \& Simões, 2015; Mourão, Albuquerque, Silva, Oliveira \& Fernandes, 2009; Santos, 2014). A comunicação não-verbal acompanha necessariamente a comunicação face-a-face, reforçando ou contradizendo o conteúdo expresso verbalmente (Martins, 2013). Todo o corpo do indivíduo comunica de forma consciente ou inconsciente. A comunicação paraverbal abrange todos os sons vocais que não são verbais, isto é, o tom e volume da voz, o ritmo da fala e a fluidez do discurso. Este tipo de comunicação pode ser classificado em dois grandes grupos: relacionados com a fala e independentes da fala (Capucho, 2003).

\subsection{Perspetivas Teóricas da Comunicação}

A raiz etimológica do substantivo comunicação está no latim "communicatione”, sendo que esta deriva da palavra "commune”, cujo significado é "comum”. Comunicar é pois "entrar em relação com”, "partilhar” (Capucho, 2003).

Pegando novamente nas fontes mais tradicionais para definição deste conceito, o substantivo comunicação pode ser definido como sendo a troca de informação/mensagens entre dois ou mais indivíduos através do uso da fala, da escrita, dos códigos comuns ou até mesmo de comportamentos. Caracteriza-se, assim, como o estabelecimento de relações com base num diálogo (Drosdowski, 1989; Hornby, 2000; Machado et al., 2014; Porto Editora, 2008; Robert, 2001).

Numa versão um pouco redutora, a comunicação caracteriza-se pelo entendimento de mensagens e troca informação entre o emissor e o recetor (Duarte, 2011). Numa visão mais alargada, o indivíduo atua sempre no sentido de criar no outro uma boa impressão (D. Gomes, 2000), isto é, o comportamento de um indivíduo que representa um papel vai no sentido de construção de uma imagem, criando impressões positivas que se pretendem transmitir naquela situação específica (Goffman, 2002).

\subsubsection{Do Modelo de Jakobson à Perspetiva Sociocomunicativa da Comunicação}

Ao longo da história foram surgindo várias teorias da comunicação, começando inicialmente como a perspetiva bastante linear de Jakobson (1969), conhecida como modelo telegráfico, passando posteriormente para uma abordagem mais complexa, desde Escola de Palo Alto, nomeadamente 
com o modelo de orquestra de Watzlawick (2011), à perspetiva de Goffman (2002).

\section{Modelo de Jakobson}

As teorias mais tradicionais apresentam como elementos da comunicação o emissor, recetor e o canal de transmissão. A comunicação apresenta fundamentalmente seis elementos (Chiavenato, 1994; Fiúza \& Kilimnik, 2004; Garcia, 2010; J. G. Neves, Garrido \& Simões, 2015; Jakobson, 1969; Ramos, 2003; Santos, 2014; Souza \& Santos, 2014; Tavares, 2011), nomeadamente:

- Emissor, codificador ou remetente - revela o indivíduo que tem uma mensagem para transmitir (com objetivos a atingir), representando a origem do processo de comunicação;

- Recetor, descodificador ou destinatário - quem recebe a mensagem, isto é, identifica quem vai reagir ao estímulo provocado pelo emissor;

- Código - conjunto de representações predefinidas entre os intervenientes no processo comunicativo que permite a compreensão das mensagens. Este combina os significantes com os significados;

- Mensagem - trata-se do que se pretende transmitir, ou seja, da informação que o emissor pretende transmitir ao recetor. É composta por um conteúdo e uma forma (maneira pela qual a mensagem é transmitida verbal ou não verbal, oral ou escrita; gestual, vocal ou espacial, etc.);

- Contacto - representado pelo canal físico e a conexão psicológica entre os indivíduos que lhes possibilita permanecer numa comunicação;

- Contexto - também designado de situação e atribui significado à mensagem entre os intervenientes. Pode ser apontado como as circunstâncias em que as mensagens são transmitidas.

Estes elementos podem por vezes confundir-se; porém, não se verifica a existência de comunicação quando um dos elementos anteriormente referidos estiver em falta (Jakobson, 1969). Para formar o ciclo simples podem ainda acrescentar-se as seguintes componentes:

- Objetivos - estímulos/interesses que o emissor tem para a transmissão da mensagem;

- Ruído - obstrução ao processo de comunicação;

- Significado - está intrinsecamente relacionado com o processo de codificação e descodificação e o código utilizado;

- Feedback - também designado de resposta, representa a reação do recetor à mensagem transmitida (Ramos, 2003; Rego, 1999; Santos, 2014). 


\section{Figura I}

Modelo básico da comunicação (Camara, Guerra \& Rodrigues, 2013;

Garcia, 2010; Ramos, 2003; Rego, 1999)

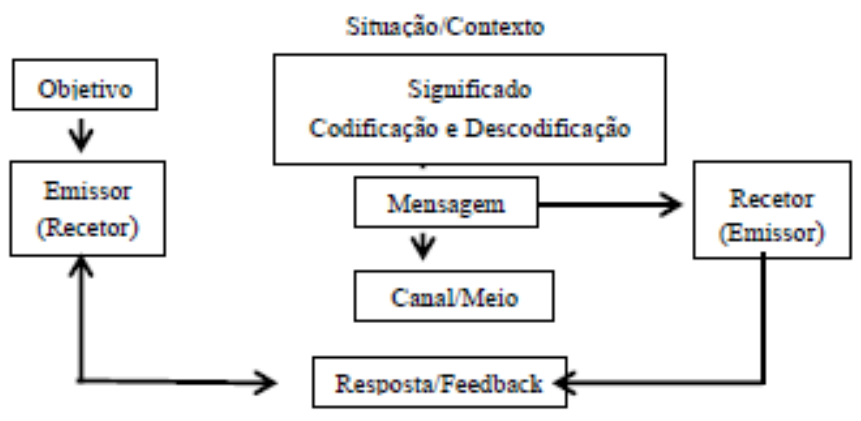

A comunicação envolve um processo de transmissão de informação, sendo que esta mesma informação é o objeto e conteúdo deste processo. A comunicação é percebida como uma forma de transmissão de informação (Jakobson, 1969). A informação é um conjunto de dados com significados específicos e os significados só existem se a informação tiver por base algum tipo de referência com os sistemas cognitivos do recetor e do emissor (Jakobson, 1969; S. S. F. Silva, Nascimento \& Nogueira, 2007). Numa troca ótima de informação, o emissor e o recetor partilham de um conjunto de conceitos e imagens comuns (Jakobson, 1969).

\section{Modelo da Escola de Palo Alto}

Numa visão mais complexa, a comunicação não é simplesmente uma expressão de mensagens, mas sim um fenómeno de interação entre um ou mais indivíduos, e que representa todos os comportamentos num contexto social. Portanto, qualquer comportamento social tem um valor comunicativo (Watzlawick, 2011).

Nesta perspetiva, a comunicação apresenta duas dimensões básicas: a construção duma relação entre os indivíduos e a transmissão de informação entre os mesmos. Ou seja, esta implica uma relação entre indivíduos e a existência de informação que se caracteriza por ser o conteúdo da mensagem. Apesar destas duas dimensões se encontrarem sempre presentes, há contextos em que uma delas se evidencia mais que a outra (Capucho, 2003). 
Comunicar é, assim, uma forma de ação sobre o outro, pois ao transmitir-se uma mensagem não se pretende apenas transmitir informação, mas também se tenciona obter um efeito sobre o outro (Watzlawick, 2011).

Quando um indivíduo tenta não comunicar com os outros que o rodeiam, está a transmitir através da sua atitude que não quer comunicar, e esta atitude por si só já é um ato comunicativo. Comunicar não é apenas uma necessidade dos indivíduos (J. S. Silva, Pinto, Rocha \& Barros, 2012), é também um imperativo da sua existência e convivência.

O conceito de feedback insere-se na noção de co-construção do discurso, pois não se trata de uma simples resposta a uma mensagem doutro indivíduo, mas sim na formação dum discurso comum com partilha de informação de todas as partes intervenientes. Esta co-construção ou cooperação, não deve ser interpretada como sinónimo de colaboração, pois não implica a ausência de conflito (Capucho, 2003).

O sentido que os interlocutores atribuem à informação que recebem depende da sua visão da realidade, e na medida em que o sentido de uma mensagem é aquele que lhe é atribuído pelo seu recetor, existe uma responsabilidade partilhada dos intervenientes no processo comunicativo. Assim surge o conceito de metacomunicação, ou seja, quando um emissor está a comunicar não está apenas a transmitir uma mensagem, mas também indica ao recetor a forma como este deverá entender a mensagem transmitida, por outras palavras, consiste na comunicação sobre comunicação (Watzlawick, 2011).

Perspetiva de Goffman: Encenação Quotidiana e Papéis dos Intervenientes

A vida em sociedade é formada por interações sociais, sendo que os papéis na sociedade são definidos por essas relações. Assim, todos os indivíduos têm a capacidade de influenciar os outros. Um indivíduo perante outros representa uma determinada imagem de si próprio, que pode ser verdadeira ou falsa, mas que é a que quer que os outros tomem como sendo a realidade, isto é, existe uma constante encenação do Eu. A face que o indivíduo conquista não depende apenas da sua representação, mas também da interpretação dos restantes indivíduos envolvidos na interação social (Goffman, 2002).

\section{Face Threatening Acts e Face Flattering Acts}

Surge então a distinção entre o que se designa de face positiva de face negativa. A primeira relaciona-se com a necessidade do indivíduo de se sentir aceite e aprovado pelos outros, enquanto a segunda vai ao encontro 
do desejo de autoafirmação, liberdade de atuação e independência (Preti, 2008). Por outras palavras, a face positiva está relacionada com imagem do indivíduo, enquanto a face negativa se refere ao território (físico ou psicológico) desse mesmo indivíduo.

Neste contexto, surgem os FTA's (Face Threatening Acts) de Brown e Levinson (1987) e FFA's (Face Flattering Acts) de Kerbrat-Orecchioni (2006). Pode ainda acontecer uma mistura dos dois casos, como por exemplo no caso de um elogio, pode-se interpretar como sendo um ato de inveja, pois quem emite um elogio está a cobiçar a coisa alheia (face negativa), mas ao mesmo tempo manifesta cortesia e simpatia (face positiva), sendo geralmente este o sentimento que prevalece (KerbratOrecchioni, 2006; Preti, 2008).

Um FTA pode ser uma ameaça à face negativa ou à face positiva para o recetor como para o emissor. Quando o emissor pressiona o destinatário numa determinada direção, isto é, invade o seu território, estamos perante uma ameaça à face negativa. Por outro lado, quando o emissor não se preocupa com sentimentos e desejos, ou seja, danifica a imagem do outro, está a emitir uma ameaça à face positiva desse indivíduo (Brown \& Levinson, 1987; Kerbrat- Orecchioni, 1992).

Concluindo, num diálogo harmonioso a emissão de um FFA leva a que a resposta seja igualmente um FFA. Por outro lado, quando um indivíduo emite um FFA em relação a outro e este responde com um FTA, ambas as partes entram em conflito.

\subsection{Formas e Barreiras Gerais à Comunicação}

A comunicação humana pode fazer-se de várias formas, sofrendo geralmente de algumas barreiras. Neste sentido, poderá afirmar-se que as formas de comunicação podem essencialmente ser agrupadas em seis categorias:

- Intrapessoal - de um indivíduo consigo próprio;

- Interpessoal - entre dois indivíduos ou entre um pequeno grupo/ grupos de pessoas, num contexto geralmente informal;

- Grupal - num grupo formal de média/grande dimensão;

- Organizacional - no seio da organização, envolvendo a comunicação interna e externa;

- Social/coletiva/de massa - em grandes grupos de indivíduos heterogéneos;

- Extrapessoal - entre as máquinas, animais ou outros seres. 
Estas formas de comunicação podem ainda ser classificadas como diretas/não mediadas - comunicação realizada sem recurso a dispositivos técnicos, como a comunicação face-a-face - ou indiretas/mediadas comunicação exercida com base em dispositivos técnicos, exemplo dos media ou livros (Sousa, 2006).

Por seu turno, as barreiras de comunicação podem ser classificadas como:

- Pessoais - relacionadas com a forma de estar, ser e agir à postura corporal;

- Físicas - obstáculos físicos entre os interlocutores;

- Culturais - relacionadas com o (des)conhecimento dos códigos de comunicação cultural;

- Psicossociais - englobando os estatutos sociais que os indivíduos envolvidos na comunicação atribuem mutuamente e que marcam uma distância social (Rego, 1999; Sousa, 2006).

Concluindo, tudo o que se interpõe entre os interlocutores e que pode distorcer a mensagem emitida pode ser classificado como uma barreira à comunicação.

\subsection{Comunicação Organizacional}

À semelhança do que acontece entre os indivíduos, a organização comunica constantemente tanto a nível interno como externo. A comunicação organizacional envolve a troca de ideias, factos, opiniões e emoções entre os elementos que a constituem, tendo duas finalidades: providenciar informação, garantido a sua correta compreensão; e proporcionar atitudes motivacionais entre os diversos elementos (S. S. F. Silva et al., 2007).

A organização acumula uma grande quantidade de informação, tanto no que diz respeito às suas operações como aos seus recursos internos (Choo, 2003). A comunicação organizacional permite que esta informação e conhecimento cheguem a todos os públicos de interesse, designados também de stakeholders - fornecedores, clientes, colaboradores, empresários, entre outros (Carneiro, 2012). Quando a comunicação organizacional se vocaciona para os colaboradores, estamos perante uma situação de comunicação interna.

\subsubsection{Importância da Comunicação para os Gestores}

Sendo que a maior parte do tempo dum gestor é despendido a comunicar com os outros (Rajhans, 2012), este deve ter habilidades 
dialógicas de forma a conseguir transmitir as mensagens corretamente, e também deve conseguir persuadir o seu público a esforçar-se na busca dos resultados desejados (Diniz, Santana \& Rodrigues, 2012). A comunicação deve ser uma tentativa de integração de todos os membros da organização, permitindo aos gestores o desempenho das suas funções, nomeadamente o planeamento, organização, liderança e controlo (Senger \& Oliveira, 2003). A linguagem do gestor deve ser de fácil compreensão, mas não deve ser demasiado simplista, pois o uso de um vocabulário demasiado vulgar pode provocar uma atitude de descrédito (Simões \& Melo, 2008).

Por fim, existem alguns atributos que podem ser melhorados pelos gestores, como a empatia, o saber ouvir os outros, o desenvolva técnicas de leitura e a capacidade de domínio da linguagem corporal. É importante notar que a velocidade da fala é superior à velocidade da compreensão, o que significa que falar rápido também é um impedimento à compreensão (Teixeira, 2005).

\subsection{Importância da Comunicação Interna}

Este tipo de comunicação tem que estimular o diálogo e a interação face-a-face entre os vários elementos da estrutura hierárquica de forma a promover os resultados desejados (J. F. Silva, 2009; Martins, 2013). Assim, a comunicação interna define-se como sendo o conjunto de técnicas utilizadas pelos gestores da organização para comunicar com o público interno, pretendendo alcançar uma maior participação de todos os elementos que constituem a organização (Duarte, 2011). Podemos considerar que os indivíduos envolvidos nos processos de comunicação interna modificam a organização e são modificados por ela (I. Oliveira \& Alencar, 2013).

A comunicação interna pode ser classificada como:

- Informativa - transmissão de informação específica sobre eventos e atividades;

- Diretiva - providência de linhas de orientação e intervenção direta de supervisores nos papéis a desempenhar;

- Interativa - na qual os colaboradores participam na troca mútua de informação;

- Indireta - utilizada quando a organização sofre mutações rápidas e necessita de uma mobilização atempada com uma utilização integral de todos os canais de comunicação (Dzamtoska, Matlievska \& Denkova, 2013). 


\subsubsection{Tipos de Comunicação Interna}

A comunicação interna pode ser dividida em comunicação oral e comunicação escrita. A comunicação oral é uma das formas muito utilizadas no interior da organização e engloba instruções, pedidos, ordens e conversas, enquanto a comunicação escrita se expressa com memorandos, cartas, cartazes, folhetos, e-mails, jornais, revistas, entre outros (Chiavenato, 1994; Santos, 2014).

A comunicação interna pode ser classificada em:

- Formal - condiz com as linhas da hierarquia formal da organização, isto é, ocorre na cadeia de comando (Camara et al., 2013; I. Oliveira \& Alencar, 2013; J. F. Silva, 2009; Machado et al., 2014; S. S. F. Silva et al., 2007; Santos, 2014). Apresenta quatro fluxos/canais básicos: descendente do topo da hierarquia à base; ascendentes - representam a resposta à informação transmitida de forma descendente; horizontal - comunicação com os pares do mesmo nível hierárquico; lateral - comunicação entre elementos de diferentes níveis hierárquicos e que não respeitem o reporte da estrutura hierárquica (Bilhim, 1996; Chiavenato, 1994; Garcia, 2010; S. S. F. Silva et al., 2007; Tavares, 2011).

- Informal - ocorre fora do contexto da estrutura organizacional de forma escrita ou oral (Chiavenato, 1994; I. Oliveira \& Alencar, 2013; S. S. F. Silva et al., 2007; Santos, 2014), e transmite a informação em todas as direções, nomeadamente: em sentido horizontal - mesmo nível hierárquico; diagonal - departamentos, funções e/ou hierarquias diferentes; gavinha em todas as direções (Carneiro, 2012; Teixeira, 2005). É frequentemente utilizada como meio de obtenção da informação que os canais formais não transmitem.

\section{A MOTIVAÇÃO}

A motivação é o ato de despertar o interesse de outro, ou seja, é o conjunto de fontes que determinam a forma de atuação de alguém, resultando numa atuação consciente. Motivar significa, pois, originar, causar, despertar interesse e fundamentar (Drosdowski, 1989; Hornby, 2000; Porto Editora, 2008; Robert, 2001; Tavares, 2011). Pode-se também associar aos conceitos de estímulo, realização, ação, objetivos, processos, atividade e indivíduo. É assim uma variável que não pode ser observada diretamente, tendo que ser inferida pelo comportamento dos indivíduos (Pasquini, Andrade, Souza \& Castro, 2005).

A motivação está ainda relacionada com a direção e intensidade de uma determinada ação, isto é, os indivíduos escolhem o sentido (direção) em 
que desenvolvem os seus esforços contínuos (intensidade) para alcançar determinados objetivos (Abbah, 2014).

Pode ser considerada como intrínseca ou extrínseca: a intrínseca referese aos comportamentos que são estimulados pela realização da atividade; a extrínseca está relacionada com todas as atividades que os indivíduos desenvolvem com a finalidade de obter uma recompensa material e/ou social ou para evitar algum tipo de punição (Cunha et al., 2007).

Os indivíduos são diferentes, com necessidades, comportamentos, valores, capacidades e objetivos distintos. Estas mesmas características variam no mesmo indivíduo conforme o tempo e o espaço em que este se encontra.

\subsection{Motivação nas Organizações}

Outrora, a única força motivacional conhecida pelos empregadores era a punição, como os cortes nos salários (Pasquini et al., 2005; S. Silva, \& Lira, 2014). Atualmente, no contexto organizacional, a motivação pode ser percebida como uma força interna estimuladora que leva os colaboradores a desenvolverem bem as suas atividades, sendo necessário perceber que cada indivíduo deste grupo tem necessidades e razões distintas dos seus semelhantes (J. S. Silva et al., 2012). Reconhece-se que os colaboradores não se satisfazem apenas com os prémios financeiros, mas também necessitam de prémios intangíveis, sendo exemplos: elogios, reconhecimento e/ou poder (Pasquini et al., 2005).

Um colaborador motivado é um colaborador leal, sendo que só se verifica lealdade se os objetivos e ações forem defendidos e aceites pelo mesmo (Rajhans, 2012). Desta forma, um colaborador motivado irá desenvolver todos os esforços necessários para atingir os objetivos organizacionais (Abbah, 2014).

Uma baixa produtividade, um grande absentismo, um incremento de produtos defeituosos, um aumento dos acidentes de trabalho ou uma menor eficiência produtiva são exemplos de que os colaboradores não se encontram motivados (Pasquini et al., 2005).

Existem vários fatores que podem ser considerados como motivadores, sobretudo: remuneração justa; subsídios e incentivos monetários; confiança e respeito; participação na tomada de decisão; qualidade na supervisão; bom ambiente de trabalho, tanto físico como social; apreciação e reconhecimento; oportunidades de crescimento; boa comunicação e acesso à informação (Abbah, 2014). 
Sintetizando, a motivação baseia-se na criação de uma relação de winwin para ambas as partes (organização e colaboradores), em que a confiança é um dos pilares bases (Abbah, 2014).

\subsection{Teorias da Motivação}

As teorias da motivação desenvolveram-se em grande medida nos anos 40, pois era necessário aumentar a eficácia e eficiência dos colaboradores e verificou-se uma mudança da perceção dos indivíduos da organização, deixando de ser vistos como meras peças (Tavares, 2011). Nas teorias da motivação existem fundamentalmente três grandes grupos:

\subsubsection{Teorias do Conteúdo}

Estas teorias relacionam-se com a satisfação das necessidades humanas. Torna-se fácil aceitar que o ser humano busque constantemente a satisfação das suas necessidades, por mais diversas e numerosas que sejam.

\section{A Teoria das Necessidades de Maslow}

Nasceu nos anos 40 e baseia-se no pressuposto da existência de necessidades insatisfeitas que motivam os indivíduos e enquanto as necessidades mais baixas não forem satisfeitas as necessidades de nível superior não se manifestam (A. L. Neves, 1998; Abbah, 2014; Bilhim, 1996; Camara et al., 2013; Chiavenato, 1990, 1993, 1994, 1995; Cunha et al., 2007; Pasquini et al., 2005; Tavares, 2011; Teixeira, 2005). As necessidades agrupam-se segundo cinco níveis de hierarquia:

- Fisiológicas - relacionadas com as necessidades de alimentação, abrigo, ar e repouso;

- De segurança - respeitantes às necessidades de proteção, ausência de violência, doenças, guerra e pobreza;

- Sociais - baseadas nas necessidades de afeição, amizade, inclusão em grupos sociais, aceitação e aprovação pelos membros do grupo social;

- De estima - fundamentadas nas necessidades de reputação, autorrespeito, admirações e reconhecimento;

- De autorrealização - apoiadas nas necessidades de realização do potencial individual.

Em síntese, as primeiras duas categorias de necessidades (necessidades fisiológicas e de segurança) designam-se ainda de necessidades primárias. As restantes três categorias (necessidades sociais, de estima e de autorrealização) assumem ainda o nome de necessidades secundárias 
(Chiavenato, 1990, 1993, 1994, 1995; J. G. Neves et al., 2015; Teixeira, 2005).

\section{A Teoria dos Dois Fatores ou Bifatorial de Herzberg}

Nascida nos anos 50, esta teoria defende que existem dois fatores importantes para o comportamento dos indivíduos:

- Fatores higiénicos - envolvem o salário, condições de trabalho, relações interpessoais, segurança, benefícios sociais, clima laboral fatores extrínsecos à atividade (fatores de contexto);

- Fatores motivacionais - incluem objetivos de realização pessoal, reconhecimento, responsabilidade, desenvolvimento, progresso, estatuto, crescimento profissional - fatores intrínsecos à atividade (fatores de conteúdo).

De certa forma, esta teoria pode ser equiparada à teoria das necessidades de Maslow, sendo que os fatores higiénicos correspondem às necessidades fisiológicas, de segurança e sociais de Maslow e os fatores motivacionais correspondem às necessidades de estima e autorrealização de Maslow. Porém, Herzberg difere de Maslow nas conclusões, isto é, os fatores higiénicos conseguem diminuir a insatisfação; porém não possibilitam motivar os indivíduos. Em contrapartida, os fatores motivacionais, tal como o nome indica, são capazes de gerar motivação nos indivíduos (A. L. Neves, 1998; Camara et al., 2013; Chiavenato, 1990, 1993, 1994, 1995; Cunha et al., 2007; J. G. Neves et al., 2015; Tavares, 2011; Teixeira, 2005).

\section{A Teoria das Necessidades de McClelland}

Esta teoria surgiu na década de 60 e baseia toda a sua atenção para as necessidades que os indivíduos adquirem ao longo da sua vida, isto é, à medida que vão interagindo com o ambiente que os circunscreve, sendo que três se tornam particularmente relevantes:

- Necessidades de realização/sucesso - relacionadas com o desejo individual de atingir desafios;

- Necessidades de poder - baseadas na aspiração de controlar, influenciar, de ser responsável por outros indivíduos e de ter prestígio;

- Necessidade de afiliação - vontade de criação e manutenção de relações pessoais com outros indivíduos;

Todos os indivíduos possuem estes três tipos de necessidades, porém existe sempre uma que se manifesta mais (A. L. Neves, 1998; Abbah, 
2014; Bilhim, 1996; Cunha et al., 2007; J. G. Neves et al., 2015; Teixeira, 2005).

\section{$\underline{\text { A Teoria ERG de Alderfer }}$}

Esta teoria remonta ao início dos anos 70 e defende que a motivação dos colaboradores pode ser explicada com base na satisfação das necessidades hierarquizadas. Porém, de acordo com esta teoria existem apenas três níveis de necessidades:

- De existência - correspondem às necessidades fisiológicas e de segurança de Maslow (em inglês designadas de Existence);

- De relacionamento - condizem com as necessidades sociais de Maslow (em inglês designadas de Relatedness);

- De crescimento - coincidem com as necessidades de estima e autorrealização de Maslow (em inglês designadas de Growth).

Segundo esta teoria, e embora na generalidade se admita que as necessidades de níveis superiores só emergem após satisfeitas as necessidades de níveis inferiores, existem situações em que isto não se verifica (A. L. Neves, 1998; Bilhim, 1996; Cunha et al., 2007; Teixeira, 2005).

\subsubsection{Teorias do Processo}

Nesta perspetiva, a motivação é analisada na forma como os indivíduos escolhem os processos para alcançarem as suas finalidades.

\section{A Teoria das Expectativas de Vroom}

Surgiu em 1964 e é também designada de modelo contingencial da motivação e pode expressar-se pela seguinte fórmula: Força da Motivação $(\mathrm{M})=$ Valência $(\mathrm{V}) \times$ Expectativa $(\mathrm{E})$

Os membros desta expressão assumem o seguinte significado:

- Valência - intensidade da preferência individual de alcançar determinado resultado;

- Expectativa - perceção da probabilidade de uma determinada ação levar ao resultado desejado;

- Força da Motivação - motivação percebida pelo colaborador (A. L. Neves, 1998; Bilhim, 1996; Chiavenato, 1990, 1994; Cunha et al., 2007; J. G. Neves et al., 2015; Santos, 2014; Teixeira, 2005).

\section{A Teoria da Equidade de Adams}

Surgiu na década de 60 e foca-se na perceção que cada indivíduo tem sobre a razoabilidade das situações laborais, comparando sempre o 
desempenho e os benefícios individuais com o desempenho e benefícios dos seus pares em situações semelhantes, isto é, encontra-se fortemente relacionada com o conceito de justiça individual (Cunha et al., 2007; J. G. Neves et al., 2015; Teixeira, 2005). Neste sentido, os indivíduos são motivados em minorar as desigualdades de tratamento percebidas (Teixeira, 2005), sendo que considera dois tipos de equidade: equidade interna (dento da organização) e externa (com o exterior da organização) (Cunha et al., 2007).

\section{A Teoria da Tarefa Enriquecida}

Esta teoria assenta fundamentalmente nos seguintes princípios:

- A tarefa deve estar estruturada de forma a que o colaborador se sinta pessoalmente responsável por uma determinada quantidade de trabalho, ou seja, deve envolver um certo grau de autonomia e responsabilidades;

- A tarefa deve ser significativa, isto é, o colaborador deve sentir que o trabalho que desempenha tem importância para a organização;

- A tarefa deve possibilitar o feedback sobre a maneira como esta está a ser executada e os resultados que estão a ser atingidos (J. G. Neves et al., 2015).

\subsubsection{Teorias do Resultado}

As teorias centram a sua atenção nos objetivos que os indivíduos pretendem alcançar

\section{A Teoria da Definição de Objetivos de Locke e Latham}

Surgiu no início dos anos 70 e defende que se existem objetivos está-se a assumir que o indivíduo em questão tem o desejo de atingir determinados resultados, sabe qual o caminho a seguir e a forma como medir a sua eficácia na execução das tarefas (Bilhim, 1996; Cunha et al., 2007; J. G. Neves et al., 2015). Quando se fixa um objetivo tem-se a crença de que um bom desempenho aumenta a possibilidade de os atingir. No interior da organização, esta teoria traduz-se normalmente num sistema de gestão por objetivos, que desempenham, em simultâneo, funções de controlo e motivação dos colaboradores (J. G. Neves et al., 2015).

\section{A Teoria do Reforço de Skinner}

Nasceu no início nos anos 70 e defende que o comportamento de um indivíduo pode ser explicado com base na previsão mais ou menos positiva/negativa das consequências desse mesmo comportamento 
(Teixeira, 2005). Neste sentido, reforço significa qualquer consequência que resulta de um dado comportamento (J. G. Neves et al., 2015). Desta forma deve-se apontar o desempenho desejado, identificar as recompensas e selecionar um plano de reforço ótimo.

\section{CONCLUSÃO}

Neste ponto passar-se-á a relacionar os três conceitos principais anteriormente abordados: organização, comunicação e motivação.

Organização e Comunicação: a organização caracteriza-se por ser um organismo, constituído por indivíduos que estabelecem relações (Pereira, 2004; Souza \& Santos, 2014; Teixeira, 2005), isto é, que comunicam entre si. Como a comunicação se baseia num processo de co-construção do discurso, os membros duma organização não se limitam a uma troca simples de mensagem, mas sim à construção dum discurso comum com partilha de informação de todas as partes intervenientes (Capucho, 2003). Cada organização tem uma determinada estrutura formal representada no seu organograma. O tipo de estrutura organizacional é relevante quando se analisa a comunicação formal (Camara et al., 2013; Machado et al., 2014; I. Oliveira \& Alencar, 2013; J. F. Silva, 2009; S. S. F. Silva et al., 2007; Santos, 2014), tanto a nível descendente (top down) como ascendente (bottom up).

Organização e Motivação: o gestor duma organização pode e deve desenvolver os esforços necessários para promover o desenvolvimento da motivação dos colaboradores (Teixeira, 2005). A capacidade de motivação numa organização representa a criação de esforços para alcançar os objetivos organizacionais gerando assim espírito de equipa.

Motivação e Comunicação: a comunicação interna com qualidade na organização é o elemento chave de todo o processo motivacional (A. L. Almeida, Baptista \& Soares, 2013), pois só é possível motivar um colaborador (criar um estímulo) se as mensagens forem transmitidas e percebidas corretamente. De notar que a comunicação tem uma forte componente relacional entre os indivíduos envolvidos (Watzlawick, 2011), permitindo criar grupos de indivíduos cooperantes entre si (ou seja, indivíduos motivados), que constituirão verdadeiras equipas de desempenho (Goffman, 2002). Note-se que entre estes termos existe um elemento comum: o feedback/resposta/retorno. 
Organização, Motivação e Comunicação: a forma como a organização comunica com os seus membros influencia tanto a moral como a sua motivação e o seu desempenho, sendo importante notar que constrói as identidades no contexto de trabalho. Assim, se os colaboradores sentirem que os seus superiores hierárquicos comunicam com eles eficientemente, terão incentivos a aumentar o seu empenho e a criar espírito de equipa (Rajhans, 2012). Por esta razão, e de forma a motivar os colaboradores para uma melhor performance, uma gestão eficiente das práticas comunicacionais internas é fundamental.

\section{FONTES E BIBLIOGRAFIA}

Abbah, M. T. (2014). Employee Motivation: The Key to Effective Organizational Management in Nigeria. IOSR Journal of Business and Management (JBM), 16 (4), 1-8. Consultado a 24 de março de 2016, em: http://www.iosrjournals.org/iosr-jbm/papers/Vol16-issue4/Version1/A016410108.pdf

Almeida, A. L., Baptista, R., \& Soares, P. H. (2013). Comunicação interna e redes sociais: desafios e possibilidades no relacionamento com os empregados. Revista Organicom, 10 (19), 72-81. Consultado a 24 de março de 2016, em:

http://revistaorganicom.org.br/sistema/index.php/organicom/article/vie w/663

Almeida, L. A. S. (2013). A importância da Comunicação Interna para a Motivação dos Colaboradores. EXEDRA Revista Científica ESEC, (8), 90-103. Consultado a 24 de março de 2016, em: http://www.exedrajournal.com/wp-content/uploads/2014/09/07.pdf

Barracho, C. (2014). Os Recursos Humanos e os Desafios da Actual Gestão: Num Contexto Global em Constante Mudança. Lusíada. Economia \& Empresa, (18), 173-184. Consultado a 24 de março de 2016, em: http://revistas.lis.ulusiada.pt/index.php/lee/article/view/1890

Bilhim, J. A. F. (1996). Teoria Organizacional: Estruturas e Pessoas. Lisboa: Instituto Superior de Ciências Sociais e Políticas.

Brown, P., \& Levinson, S. C. (1987). Politeness: some universals in language usage. Cambridge: Cambridge University Press.

Burns, T., \& Stalker, G. M. (1961). The Management of Innovation. Em Tosi, H. L. (Eds.). Theories of Organization (pp. 103-108). EUA: SAGE Publications Ltd.

Camara, P. B. da, Guerra, P. B., \& Rodrigues, J. V. (2013). Humanator XXI: Recursos Humanos e Sucesso Empresarial (6 ${ }^{\mathrm{a}}$ ed.). Alfragide: Publicações Dom Quixote. 
Capucho, M. F. (2003). Communication verbale et non-verbale. Em Maigret, E. (Eds.). Communication et Médias (pp. 11 - 15). Paris: Les notices - La documentation Française.

Carneiro, L. A. (2012). A comunicação interna para promoção da qualidade. E-Locução - Revista Científica da FAEX, 1 (1), 38-53. Consultado a 24 de março de 2016, em:

http://www.faex.edu.br/periodicos/index.php/e-locucao/article/view/5

Chiavenato, I. (1990). Recursos Humanos (10 triagem). Edição compactada. São Paulo: Editora Atlas S.A.

Chiavenato, I. (1993). Introdução à Teoria Geral da Administração (4 ed.). São Paulo: Makron Books.

Chiavenato, I. (1994). Administração: Teoria, Processo e Prática (2ª ed.). São Paulo: Makron Books.

Chiavenato, I. (1995). Administração de Empresas: uma abordagem contingencial ( $3^{\mathrm{a}}$ ed.). São Paulo: Makron Books.

Choo, C. W. (2003). Gestão da informação para organização inteligente: a arte de explorar o meio ambiente (A. Fonseca, Trad.). Lisboa: Editorial Caminho (Obra original publicada em 1998).

Cunha, M., Rego, A., Cunha, R., \& Cabral-Cardoso, C. (2007). Manual de Comportamento Organizacional e Gestão ( 6 a ed.). Lisboa: RH Editora.

DeStefano, M. (2013). Creating a Culture of Compliance: Why Departmentalization May Not Be the Answer. Hastings Business Law Journal, 10, 71-182. Consultado a 24 de março de 2016, em: http://papers.ssrn.com/sol3/papers.cfm?abstract_id=2391434\#\#

Diniz, A. Q., Santana, D. T. de, \& Rodrigues, M. C. (2012). Comunicação organizacional. $2^{\circ}$ Congresso de Pesquisa Científica: Inovação, Ética e Sustentabilidade, 26-32. Consultado a 24 de março de 2016, em: http://revista.univem.edu.br/index.php/REGRAD/article/viewArticle/427

Drosdowski, G. (1989). Duden Deutsches Universalwörterbuch A-Z. Mannheim: Dudenverlag.

Duarte, G. (2011). Dicionário de administração. Fortaleza: Imprensa Universitária: IMPARH.

Dzamtoska, S., Matlievska, M., \& Denkova, J. (2013). Internal communication as a precondition for successful management: theoretical approach. (JPMNT) Journal of Process Management - New Technologies, Internationa, 1 (2), 96-10.

Ferreira, A. I., Martinez, L. F., Nunes, F. G., \& Duarte, H. (2015). Gestão de Recursos Humanos para Gestores ( $1^{\mathrm{a}}$ ed.). Lisboa: Editora RH.

Firmino, M. B. (2002). Gestão das Organizações: Conceitos e Tendências Actuais. Lisboa: Escolar Editora. 
Fiúza, M. S. S. F., \& Kilimnik, Z. M. (2004). Indicadores de mensuração da qualidade de comunicação : estudo de caso em Instituição de Ensino Superior. Revista Administração On Line - FECAP, 5 (2), 1-20. Consultado a 24 de março de 2016, em: http://www.fecap.br/adm_online/art0502/art5021.pdf

Francisco, L. A. (2012). Comunicação e Serviço de Referência: aspectos semióticos e tecnológicos. Revista Brasileira de Biblioteconomia e Documentação - RBBD, 9 (1), 2-16. Consultado a 24 de março de 2016, em: http://rbbd.febab.org.br/rbbd/article/view/184

Garcia, A. (2010). A contribuição da comunicação interna para a motivação dos colaboradores de uma organização: estudo de caso XP investimentos matriz Rio de Janeiro/RJ. Trabalho Bacharelato em Comunicação Social, Escola Superior de Propaganda e Marketin, Porto Alegre. Consultado a 24 de março de 2016, em: http://endomarketing.com/wp-content/uploads/2014/10/publicacaomonografia-amanda-silva-garcia.pdf

Goffman, E. (2002). A Representação do Eu na Vida Cotidiana (10ª ed., M. C. S. Raposo, Trad.). Rui de Janeiro: Editora Vozes (Obra original publicada em 1959).

Gomes, D. (2000). Cultura Organizacional comunicação e identidade. Coimbra: Quarteto Editora.

Gomes, S. (2014). Estilos de Liderança dos Diretores Técnicos e Satisfação dos Colaboradores - Estudo realizado em IPSS`s dos Distritos da Guarda e Viseu. Dissertação de Mestrado em Gestão, especialização em Gestão de Recursos Humanos, Universidade Católica Portuguesa, Centro Regional das Beiras, Viseu.

Hornby, A. S. (2000). Oxford Advanced Learner's Dictionary (6 $6^{\mathrm{a}}$ ed.). Oxford: OXFORD University Press.

Jakobson, R. (1969). Lingüística e Comunicação. São Paulo: Editora Cultrix.

Kerbrat- Orecchioni, C. (1992). Les interactions verbales, tome II. Paris: Armand Colin.

Kerbrat- Orecchioni, C. (2006). Análise da conversação. Princípios e Métodos. São Paulo: Parábola Editorial.

Kich, J. I., \& Pereira, M. (2011). A influência da liderança, cultura, estrutura e comunicação organizacional no processo de implantação do planejamento estratégico. Cadernos EBAPE.BR, 9 (4), 1045-1065. Consultado a 24 de março de 2016, em:

http://bibliotecadigital.fgv.br/ojs/index.php/cadernosebape/article/view/ 5233/3967 
Machado, A. R., Machado, D. M. R., \& Portugal, M. N. (2014). Organizações - Introdução à Gestão e Desenvolvimento das Pessoas. Lisboa: Escolar Editora.

Martins, M. T. (2013). Diálogo e interações face a face: a força da oralidade na comunicação interna. Revista Organicom, 10 (19), 194208. Consultado a 24 de março de 2016, em:

http://revistaorganicom.org.br/sistema/index.php/organicom/article/vie $\mathrm{w} / 650$

Mourão, C. M. L., Albuquerque, A. M. S., Silva, A. P. da, Oliveira, M. S. de, \& Fernandes, A. F. C. (2009). Comunicação em Enfermagem: uma revisão bibliográfica. Revista Rene. Fortaleza, 10 (3),139-145. Consultado a 24 de março de 2016, em: http://www.revistarene.ufc.br/vol10n3_pdf/a17v10n3.pdf

Nascimento, A., Marinho, E., Ramos, M., Santos, R., Guimarães, R., \& Menezes, M. (2014). O desafio do planejamento de Recursos Humanos ante as novas demandas do mercado. Cadernos de Graduação Ciências Humanas e Sociais, 2 (1), 13-24. Consultado a 24 de março de 2016, em:

https://periodicos.set.edu.br/index.php/cadernohumanas/article/view/986

Neves, A. L. (1998). Motivação para o trabalho: dos conceitos às aplicações. Lisboa: Editora RH.

Neves, J. G. das, Garrido, M. V., \& Simões, E. (2015). Manual de Competências Pessoais, Interpessoais e Instrumentais: Teoria $e$ Prática ( $3^{\mathrm{a}}$ ed.). Lisboa: Edições Sílabo.

Oliveira, I., \& Alencar, T. G. (2013). A dinâmica comunicativa no ambiente interno das organizações: inter-relação da comunicação formal com a comunicação informal. Revista Organicom, 10 (19), 209219. Consultado a 24 de março de 2016, em: http://revistaorganicom.org.br/sistema/index.php/organicom/article/vie $\mathrm{w} / 649$

Oliveira, J. T. de (2014). Mudanças na estrutura organizacional de empresas que alteram significativamente seus modelos de negócios. Tese Doutoramento em Administração, Universidade Municipal de São Caetano do Sul, São Caetano do Sul. Consultado a 24 de março de 2016, em: http://repositorio.uscs.edu.br/handle/123456789/606

Pasquini, A. O., Andrade, F. A. de, Souza, F. G. de, \& Castro, M. O. de (2005). Motivação no trabalho: um estudo no supermercado Pastorinho S/A. Trabalho de Bacharelato em Administração, Faculdade de Ciência Económicas e Administrativas de presidente Prudente, São Paulo. Consultado a 24 de março de 2016, em: 
http://intertemas.unitoledo.br/revista/index.php/Juridica/article/view/39 $7 / 392$

Pereira, O. G. (2004). Fundamentos de Comportamento Organizacional ( $2^{\mathrm{a}}$ ed.). Lisboa: Fundação Calouste Gulbenkian.

Pinto, A. T. F. M. (2013). A Eficácia da Comunicação Organizacional: Estudo Aplicado à Associação Empresarial AIRV. Projeto de Mestrado em Comunicação e Marketing, IPV - Escola Superior de Educação de Viseu, Viseu.

Porto Editora (2008). Dicionário da Língua Portuguesa. Porto: Autor.

Preti, D. (2008). Cortesia verbal. São Paulo: Humanitas. Consultado a 24 de março de 2016, em:

https://books.google.pt/books?id=FH0VD44wnLgC\&pg=PA312\&lpg= PA312\&dq=FTA+E+FFA\&source=bl\&ots=F7mcZvZIdJ\&sig=TKmeT pe8xc_gmbOeGyHBeoOLy_k\&hl=pt-

PT\&sa=X\&ved=0ahUKEwjDrY_Mu_rJAhWEXRoKHZm3CusQ6AEI HTAA\# $=$ =nepage \&q=FTA\%20E\%20FFA\&f=false

Rajhans, K. (2012). Effective Organizational Communication: a Key to Employee Motivation and Performance. Interscience Management Review (IMR), 2 (2), 81-85. Consultado a 24 de março de 2016, em: http://interscience.in/imr_vol2iss2/imr_paper16.pdf

Ramos, C. L. (2003). Barreiras e estímulos da comunicação interpessoal nas organizações. Monografia de curso de Psicologia, UniCEUB Centro Universitário de Brasília, Brasília. Consultado a 24 de março de 2016, em: http://repositorio.uniceub.br/bitstream/123456789/2843/2/9907500.pdf Rego, A. (1999). Comunicação nas Organizações Teoria e Práticas (1 ed). Lisboa: Edições Sílabo.

Robert, P. (2001). Le Nouveau Petit Robert: Dictionnaire De La Langue Francais. Paris: Dicionnaires Le Robert.

Santos, C. I. F. dos (2014). A comunicação com a equipa: que procedimentos adoptar para aumentar a produtividade. Monografia de Mestrado em Ciências Farmacêuticas, Universidade Lusófona de Humanidades e Tecnologias, Lisboa.

Schelles, S. (2008). A importância da linguagem não-verbal nas relações de liderança nas organizações. Revista Esfera, (1). Consultado a 24 de março de 2016, em:

http://www.institutoidentidade.com.br/fotos/artigospdf/A\%20importanc ia\%20da\%20comunicacao\%20nas\%20rela\%C3\%A7\%C3\%B5es\%20de \%20lideran\%C3\%A7a.pdf

Senger, I., \& Oliveira, L. C. F. (2003). Comunicação organizacional: um meio de integração e envolvimento dos agentes nos ambientes 
organizacionais. Revista de Administração 2 (3), 111-132. Consultado a 24 de março de 2016, em:

http://revistas.fw.uri.br/index.php/revistadeadm/article/view/762

Silva, J. F. (2009). A comunicação interna e a imagem organizacional. Estudo de caso na Fnac Braga. Dissertação de Mestrado em Ciências da Comunicação, especialização em Relações Públicas e Publicidade, Instituto de Ciências Sociais da Universidade do Minho, Braga.

Silva, J. S. da, Pinto, F. R., Rocha, J. D., \& Barros, C. M. P. (2012). A relação entre comunicação organizacional interna e motivação: um estudo de caso numa instituição de ensino superior. Revista Expectativa - Unioeste, 11 (11), 123 -143. Consultado a 24 de março de 2016, em: http://e-revista.unioeste.br/index.php/expectativa/article/view/7278

Silva, S. S. F. da, Nascimento, T. C. C., \& Nogueira, V. B. (2007). Diagnóstico da comunicação interna e desenvolvimento de um plano integrado de comunicação empresarial - PICE no Hospital Regional de PICUI - PB. QUALIT@S Revista Eletrônica, 6, (1). Consultado a 24 de março de 2016, em:

http://revista.uepb.edu.br/index.php/qualitas/article/viewFile/95/76

Silva, S., \& Lira, R. A. (2014). Endomarketing como ferramenta de motivação: análise das suas aplicações em uma rede de supermercados no município de Campos Goytacazes - RJ. Revista Cientifica Perspectivas Online, 4 (9), 35-46. Consultado a 24 de março de 2016, em:_http://www.seer.perspectivasonline.com.br/index.php/humanas_so ciais_e_aplicadas/article/view/148/475

Simões, D. M. P., \& Melo, E. M. de (2008). Pensamento, organização e clareza: comunicação e liderança. Revista Universitas, (1), 9-22. Consultado a 24 de março de 2016, em:

http://www.revistauniversitas.inf.br/index.php/UNIVERSITAS/article/v iew/101

Sousa, J. P. (2006). Elementos de Teoria e Pesquisa da Comunicação e dos Media ( $2^{\mathrm{a}}$ ed.). Consultado a 24 de março de 2016, em: http://www.bocc.ubi.pt/pag/sousa-jorge-pedro-elementos-teoriapequisa-comunicacao-media.pdf

Souza, R., \& Santos, J. (2014). Comunicação Interna: uma contribuição para a melhoria das relações de trabalho. Revista Gestão \& Tecnologia, 14, (2), 180-199. Consultado a 24 de março de 2016, em: https://revistagt.fpl.edu.br/get/article/view/422/539

Tavares, E. C. M. (2011). Comunicação interna como agente de motivação dos colaboradores - estudo de caso: agência nacional das comunicações. Monográfica de licenciatura em Relações Públicas e 
Secretariado Executivo, Escola de Negócios e Governação, Praia. Consultado a 24 de março de 2016, em:

http://www.portaldoconhecimento.gov.cv/bitstream/10961/231/1/Junho \%202011\%20ELIS\%C3\%82NGELA\%20CRISTINA\%20MENDES\%2 0TAVARES\%20.pdf

Teixeira, S. (2005). Gestão das Organizações (2a ed). Espanha: McGraw Hill.

Vanderley, L. (2001). Capital Humano: a Vantagem Competitiva. Organizações \& Sociedade (O\&S), 8 (22), 65-74. Consultado a 24 de março de 2016, em:

http://www.portalseer.ufba.br/index.php/revistaoes/article/viewArticle/ 10584

Watzlawick, P. (2011). Münchenhausens Zopf oder : Psychtherapie und «Wirklichkeit» (2 $2^{\mathrm{a}}$ ed.). Bern: Verlag Hans Huber. 